\title{
A Practical Solution to the Client-LDNS Mismatch Problem
}

\author{
Cheng Huang \\ Microsoft Research
}

\author{
Ivan Batanov \\ Microsoft Corporation
}

\author{
Jin Li \\ Microsoft Research
}

\begin{abstract}
Internet services are often deployed in multiple (tens to hundreds) of geographically distributed data centers. They rely on Global Traffic Management (GTM) solutions to direct clients to the optimal data center based on a number of criteria like network performance, geographic location, availability, etc.

The GTM solutions, however, have a fundamental design limitation in their ability to accurately map clients to data centers - they use the IP address of the local DNS resolver (LDNS) used by a client as a proxy for the true client identity, which in some cases causes suboptimal performance. This issue is known as the client-LDNS mismatch problem. We argue that recent proposals to address the problem suffer from serious limitations. We then propose a simple new solution, named "FQDN extension", which can solve the clientLDNS mismatch problem completely. We build a prototype system and demonstrate the effectiveness of the proposed solution. Using JavaScript, the solution can be deployed immediately for some online services, such as Web search, without modifying either client or local resolver.
\end{abstract}

\section{Categories and Subject Descriptors}

C.2.2 [Computer-Communication Networks]: Network Protocols

\section{General Terms}

Design, Performance

\section{Keywords}

Latency, DNS, GTM

\section{INTRODUCTION}

Internet services are often deployed in multiple - tens to hundreds of geographically distributed locations, so that they can be close and deliver fast and responsive services to clients. For example, Amazon's AWS are deployed to data centers in 18 locations with major network infrastructures over 3 continents [1]. Google is reported to have deployed its search services to 36 data centers worldwide [2]. Akamai's content distribution network is estimated to have footprint in hundreds of locations [3].

To optimize client performance, the best data center needs to be dynamically determined for each individual user. By serving the users from the best data center, content such as web search results, Internet videos, online gaming messages and software updates can be delivered with lowest latency and highest throughput, while causing least amount of congestion on network backbones at the same time.

Empirical evidences have shown that the best data center correlates poorly with geographic distance. Instead, it is determined by a combination of network latency, packet loss and available bandwidth. In addition, the best data center is dynamic, which changes in time, as Internet routes flap, ISPs relationships are modified, or the connectivity of physical networks fluctuates, and so on. Dynamically and accurately determining the best data center is the cornerstone of a Global Traffic Management (GTM) solution. Here, the key challenge is to accurately locate the best data center for each client on a continuous basis.

GTM solutions are often implemented through DNS systems. Say, a particular service, deployed to many data centers over several continents, is accessible via www . service.com. Before accessing the service, the client first needs to resolve the hostname www.service.com and obtain the corresponding IP address. During this DNS resolution process, the client sends a DNS query to resolve the hostname. The query is handled by the authoritative name server of the domain service.com, which also runs a GTM solution. The GTM examines the source IP address of the query, determines the best data center based on the source IP address of the query, and returns the IP address of the selected "best" data center. The client then connects to the service using the returned IP.

\subsection{The Client-LDNS Mismatch Problem}

In practice, the client never directly sends DNS queries to authoritative name servers. Rather, it submits the DNS query to a local DNS resolver (LDNS), which often times is operated by the client's ISP. It is the LDNS that ultimately interacts with the authoritative name servers. As soon as the LDNS receives an answer from the authoritative name server, it forwards the answer to the client. In addition, the LDNS caches the answer so that following queries of the same hostname can be answered immediately. (DNS responses are cached for their respective Time-to-live (TTL), which is specified in the answer itself.) The LDNS caching avoids unnecessary DNS queries against the authoritative name server and speeds up the DNS resolution process, which ultimately improves the response time of the service.

LDNS, however, creates a problem for GTM - when the authoritative name server receives a DNS query, the GTM only observes the origin (IP address) of the LDNS, rather than that of the client. Therefore, the best data center determined by the GTM is in fact based solely on the LDNS, 


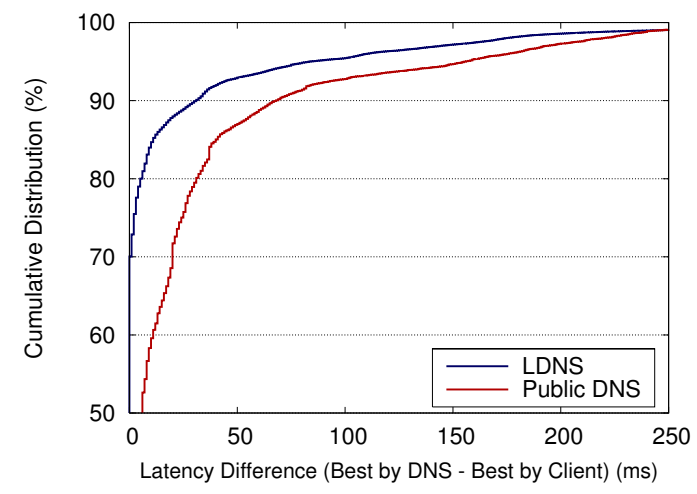

Figure 1: The Client-LDNS Mismatch Problem. Over $7.5 \%$ of Bing clients are subject to latency inflation of $50 \mathrm{~ms}$ or more. The percentage increases to $14 \%$ when clients use public DNS systems (instead of ISP-based LDNS).

instead of the client. In other words, the GTM is forced to use the LDNS as an approximation of the client.

Clearly, if the LDNS and the client were in the same location and connected from the same physical network, the LDNS would have been a reasonable approximation of the client. The reality, unfortunately, does not side with such assumption.

Indeed, as pointed out by studies dated back to circa 2000, the Internet paths to clients and their LDNS were typically disjoint and could differ 8 hops or more [4]. Also, only $64 \%$ of the clients shared the same AS-cluster with their LDNS, and the ratio dropped to $16 \%$ in network-aware clustering [5]. Sadly, the situation hasn't improved during the decade. As reported in our recent study (shown in Figure 1) [6], based on a large population of Bing clients and 17 Microsoft data centers, more than $7.5 \%$ of all the clients would be directed to sub-optimal data centers and therefore experience latency inflation of $50 \mathrm{~ms}$ or more - for every single $\mathrm{RTT}^{1}$ - when the best data centers is chosen based on LDNS.

Moreover, our measurement shows that replacing ISPbased LDNS with public DNS systems is gaining traction quickly (see Section 4.4), which tends to make the matter worse - the percentage of clients subject to latency inflation increases to $14 \%$ using public DNS systems [6]. ${ }^{2}$

In the Internet services era when $100 \mathrm{~ms}$ extra delay can cost $1 \%$ drop in sales [7], significant efforts have been invested in engineering faster Web browsers, optimizing network protocols [15], speeding up middle miles [16] and reducing round trips [17]. Complementing these efforts, solving the decade-long client-LDNS mismatch problem now appears a very fruitful area to further reduce latency.

\subsection{Key Idea and Contributions}

In this paper, we propose a simple new solution, named "FQDN extension" to address the client-LDNS mismatch problem. The key idea is to make a simple modification to hostnames so that they carry augmented information about clients to facilitate data center selection. The contributions of this paper are as follows:

1. We first survey existing proposals to address the client-

\footnotetext{
${ }^{1}$ A typical Web search response takes 3-4 RTTs.

${ }^{2}$ The degradation due to public DNS systems has also been observed by other studies $[19,20]$.
}

LDNS mismatch problem, including those that change Internet services to re-write URLs for individual clients, those that switch clients from their LDNS to public DNS systems, and those that modify LDNS to pass client-specific information. We discuss the limitations of these existing proposals.

2. We then propose a new FQDN extension solution, which makes a simple modification to hostnames so that they carry information about clients to facilitate data center selection. The key idea is to dynamically cluster clients together and assign an identifier, called GTM ID, to each cluster. The clients then augment the hostnames with the GTM IDs. When GTM receives the augmented hostnames, they are able to select the best data center based on the GTM IDs and therefore bypass LDNS completely.

3. We implement the FQDN extension in a prototype GTM system and compare with two production GTM systems based on anycast. Using PlanetLab nodes as clients, our experiments load a $17 \mathrm{~KB}$ object from the best one selected among 17 Microsoft data centers, by various GTMs. The results show that the proposed solution can reduce the object load time by as much as $46 \%$ at the 95 -percentile. We then experiment an adversary client-LDNS mismatch scenario by forcing all the clients to use public DNS systems. While the performance further degrades with the production GTMs, the performance is resilient and unaffected at all with the proposed solution.

\section{OVERVIEW OF EXISTING SOLUTIONS}

In this section, we review prior attempts to solve the client-LDNS mismatch problem and point out their limitations.

\subsection{Server-Side URL Rewriting}

Server-side URL-rewriting is a late-binding process beyond the DNS resolution stage. Due to the client-LDNS mismatch problem, the client might connect to a suboptimal data center for the first time. However, once a service observes the client's IP address, it is able to consult GTM and determine the best data center for the client. Then, the service modifies all the URLs of a returning webpage so that these URLs point to the best data center using its IP address directly. In this way, the following requests from the client can be routed correctly to the best data center.

Unfortunately, server-side URL-rewriting requires tight integration of GTM with HTTP servers. Compared to serverside URL-rewriting, DNS-based GTM solutions can be completely decoupled from the service and independent of the application protocol used. The decoupled architecture is much more flexible, where both GTM and the service can be independently developed, managed and operated. Therefore, except for very few tightly integrated systems (YouTube being one example [14]), DNS-based GTM solutions are almost exclusively adopted.

\subsection{Public DNS System}

To address the client-LDNS mismatch problem, a cloud service provider could offer its own DNS system to the public. For example, by offering Google Public DNS, any client that accesses Google's services and uses Google Public DNS sends all DNS queries to the Google Public DNS system. Because the Google Public DNS system has the knowledge of all Google data centers, and is able to observe the IP address of the client from the DNS queries, it can therefore 
select the best data center for the client based on the IP address.

Public DNS systems, however, can become a problem for third party service providers. Our recent study shows that the servers of three public DNS systems are not as widely deployed as the LDNS from Internet Service Providers [6]. Therefore, from the perspective of third party services, using the public DNS servers as an approximation of the clients appears to be even worse than using the LDNS from ISPs. Public DNS systems will not be able to optimize for a generic service provider, as it does not have information of the service provider's data center locations and network connectivity.

\subsection{Modifying LDNS}

Another recently emerged proposal to address the clientLDNS mismatch problem is to modify LDNS server software. Because the LDNS receives the DNS query directly from a client, it obviously can observe the client IP address. Based on the proposed modification, when the LDNS sends the DNS query to the authoritative name server, it attaches the prefix (of a specified length) of the client IP address together with the query. That way, the authoritative name server can select the best data center based on the address prefix, rather than the address of the LDNS [8]. The prefix is used so that following queries from other clients with the same prefix can be answered immediately from the LDNS cache.

The proposal is currently being considered at IETF. Yet, the success of the proposal hinges on the adoption and implementation of this protocol change on hundreds of thousands of LDNS servers worldwide, which requires the cooperation from thousands of ISPs, small and large. Historically, such adoption process has been quite slow.

\section{THE FQDN EXTENSION SOLUTION}

We summarize the requirements that an ideal solution to the client-LDNS mismatch problem should satisfy, which are

1. It should be based on DNS infrastructure;

2. It does not require any changes to LDNS;

3. It is efficient in utilizing LDNS cache;

4. It is accurate and flexible for all services, no matter how many data centers they are deployed in.

Next, we will describe a novel proposal to augment existing DNS-based GTM solutions. The proposed solution, therefore named "FQDN extension", satisfies all the above requirements.

\subsection{The Key Idea}

The key idea of our proposal is to modify hostnames and augment them with certain identifiers (called GTM IDs) before performing DNS resolution. GTM IDs are clientdependent, but are shared among many clients. It suffices as long as GTM IDs carry enough information about clients such that the best data center can be selected based solely on the GTM IDs. For example, if all clients from a certain prefix map to one single best data center, then the same GTM ID - possibly an encoded string of the prefix - can be assigned to all the clients. Network-aware clustering of clients is a well-explored topic and already adopted in production [10], which can be leveraged for GTM assignment.

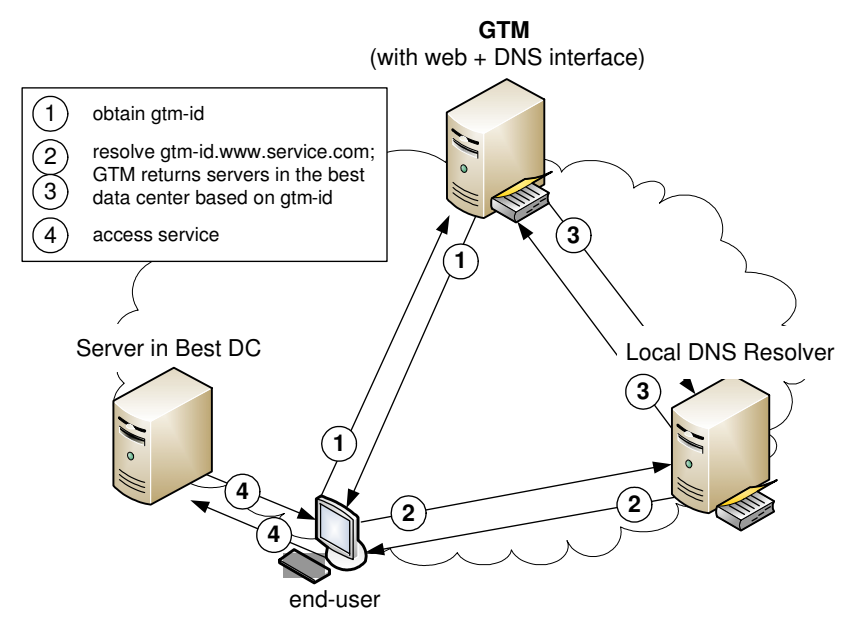

Figure 2: The FQDN Extension Solution

The modified hostnames now carry sufficient information about the clients, which allows GTM to make optimal data center selection. During DNS resolution, GTM uses the GTM IDs embedded in the hostnames to select the best data centers. Because the GTM IDs carry enough information about clients, Global Traffic Management solutions no longer need to use the LDNSes as an approximation of the clients. Therefore, the client-LDNS mismatch problem is solved.

Clearly, the proposal is a DNS-based solution and does not require any changes to LDNS. It is also efficient in utilizing LDNS cache. For example, all the clients connecting to a single Point of Presence (POP) should be aggregated into a single GTM ID. Because a hostname is always augmented with the same GTM ID, an early DNS query cached by LDNS can benefit subsequent queries from the same PoP. Of course, as different PoPs could now be assigned with different GTM IDs, it is therefore conceivable that the proposed solution will incur more cache miss on LDNS, create more entries for LDNS to cache and increase the load on GTM. Nevertheless, it still leverages the great caching capability of the DNS infrastructure. Otherwise, the client-LDNS problem can be trivially solved by having each client acting as its own LDNS.

In addition, depending on the footprint of a Internet service, a GTM ID can be assigned to a large or small client population. Intuitively, if the service is deployed in very few (say 2 or 3) data centers worldwide, one single GTM ID can be assigned to a large number of clients, as these clients can be aggregated together from the perspective of global traffic management. Conversely, if the service is deployed in hundreds of data centers, one single GTM ID is likely to be assigned only to a small number of clients, perhaps even smaller than a / 24 prefix when necessary.

\subsection{Detailed Process}

The detailed process of the FQDN extension solution is illustrated in Figure 2 and elaborated in the following steps:

- Step 0: A GTM solution offers a programmable interface (such as a web interface) so that any client may query and obtain a GTM ID.

- Step 1: A client sends a request to the GTM inter- 
face to retrieve a GTM ID for a given domain, say service.com. The GTM observes the IP address of the client and determines a GTM ID (denoted as gtm-id) for the client and the specified domain. The client caches the GTM ID within the expiration period specified by the GTM.

Note that the GTM ID only carries information about the client. Its purpose is to designate which clients should be aggregated together. The GTM ID should not carry the information of the best data center for the client, which is only dynamically determined upon DNS resolution by also taking into account other factors such as load and cost, etc.. Therefore, GTM IDs are relatively stable and adopt a much longer expiration period than the TTLs of DNS responses.

- Step 2: When the client visits any URL in the domain, say http://www . service.com/index.html, it (locally) augments the hostname with the GTM ID. In this case, the modified hostname becomes gtm-id.www. service.com.

- Step 3: The client sends a DNS query of gtm-id.www . service. com to its LDNS, which forwards the query as normal to the authoritative name server of service.com. The name server, running the GTM solution, examines the gtm-id, determines the best data center at the moment for clients grouped by the gtm-id, and responds to the DNS query with the selected data center.

- Step 4: The client connects to the data center to access the service.

\subsection{Generalization}

GTM needs to constantly adapt data center selections based on dynamic network conditions. This requires continuous monitoring and updating the performance metrics between all clients and each of a service's data centers.

The conventional approach is to create active traffic to collect measurements. For example, clients are randomly selected and redirected to suboptimal data centers from time to time $[9,10,13]$. Sniffing traffic captured at the data centers can then be used to calculate network performance. Clearly, only a small fraction of clients are selected to probe the suboptimal data centers. Nevertheless, the clients being chosen are unfortunate as they inevitably suffer performance degradation.

Even after aggregating clients with similar performance into same GTM IDs, we foresee that there will be hundreds of thousands of GTM IDs that GTM would have to keep track of. Alternatively, it is possible for GTM to enlist help from ISPs. Intuitively, ISPs have much better knowledge about what clients can be aggregated together and it is much also easier for them to keep track of the performance between their clients to the data centers.

Therefore, we propose a generalization of the FQDN extension scheme, which is to delegate GTM ID assignment to the ISPs (or any authorities for the clients) ${ }^{3}$ Now, the GTM IDs will contain not only the information about the clients, but also the information about the authorities. Imagine the GTM IDs now also include GTM_AUTH_IDs. Whenever GTM receives a query, it can use the GTM_AUTH_ID to

\footnotetext{
${ }^{3}$ Enlisting provider help for distance information has been explored in [22].
}

discover the authority. Then, GTM can query the authority with the GTM IDs and obtain the best data center selection.

Finally, when the service is delivered through Content Distribution Network (CDN), it might make sense to delegate GTM ID assignment to the CDN vendor. Of course, this requires close cooperation between the cloud provider and the $\mathrm{CDN}$ vendor.

\section{EVALUATION}

\subsection{Prototype System}

To evaluate the FQDN extension solution, we have built a simple prototype GTM system. The GTM system consists of a web server and a DNS server to serve the domain fqdnext.msrapollo.net. Any client can query the web server and obtain a GTM ID. For simplicity, the GTM system assumes all clients behind the same / 24 prefix can be aggregated into one single cluster and simply returns an encoded string of the prefix. For example, a client from 192.168.0.1 gets gtmid-COA80000, which simply comes from converting each octet of the prefix into a hexadecimal number and then concatenate the hexadecimal numbers together. ${ }^{4}$ When resolving a hostname (say www. fqdnext.msrapollo.net), the client uses the augmented hostname gtmid-COA80000. www. fqdnext.msrapollo.net instead. The GTM system converts the GTM ID back to the prefix and selects the best data center in the Microsoft global data center network.

\subsection{Experiment Setup}

We use PlanetLab nodes as clients. This makes performance data collection simple - the GTM system simply sends Ping probes from each of the data centers to all the PlanetLab clients and uses the minimum Ping latency to determine the best data center for each client. Our client population consists of 207 lightly loaded PlanetLab nodes, each from a different PlanetLab cluster.

We use a $17 \mathrm{~KB}$ image as our test object, which is about the same size as a typical web search response. The test object is placed on all the data centers. Each client loads the test object from the best data center selected by the GTM and measures the load time. Each experiment is repeated four times and the very first one is discarded to discount the DNS resolution time, as our prototype GTM system implementation is not optimized.

\subsubsection{Production GTMs for Comparison}

For comparison purpose, each client also loads the test object from the best data center determined by two production GTM systems. The first production GTM system deploys one DNS server in every data center. All the DNS servers announce the same anycast [12] IP address for a particular domain. When a client resolves a hostname in the domain, the DNS query is routed to the anycast-closest DNS server, which then returns the IP address of its local data center. This is a popular solution also adopted by many large Internet services, such as Windows Azure, Limelight [3] and etc..

Note that the anycast-closest is affected by the origin of the DNS query - that is, the LDNS of the client. Because the system uses DNS-based anycast, we call it Anycast GTM by DNS.

\footnotetext{
${ }^{4}$ Practical systems should implement better client-clustering methods $[10,21]$ and would certainly be more complicated.
} 


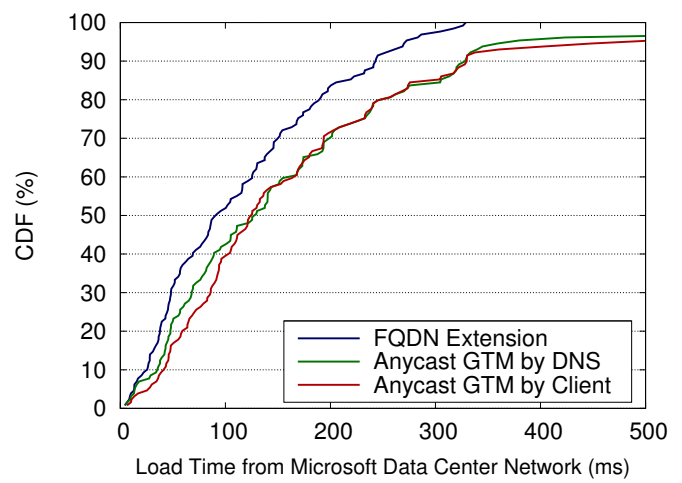

Figure 3: FQDN Ext. vs. Production GTMs

The second production GTM system is specifically designed to address the client-LDNS problem. It always returns the same anycast IP address to any DNS queries, and the anycast IP address is announced from all the data centers. In this case, a client naturally connects to the anycastclosest data center determined by its own IP, which is not affected by its LDNS at all. We call it Anycast GTM by Client.

\subsection{Comparison Results}

The performance of loading the $17 \mathrm{~KB}$ test object on all the 207 PlanetLab nodes is shown in Figure 3. We observe that our prototype GTM system based on FQDN extension significantly outperforms the two production GTM systems. The median load time is $92 \mathrm{~ms}$ for FQDN extension, compared to $129 \mathrm{~ms}$ and $126 \mathrm{~ms}$ for the production GTM systems. At the 95-percentile, the load time is $273 \mathrm{~ms}$ for FQDN extension, compared to $380 \mathrm{~ms}$ and $507 \mathrm{~ms}$ for the production GTM systems - a reduction of $28 \%$ and $46 \%$, respectively!

From the comparison, it is clear that anycast-based approaches are not effective in addressing the client-LDNS mismatch problem. Even as the Anycast GTM by Client completely bypasses LDNS, anycast itself still leads to suboptimal data center selection. This has also been reported by a number of previous studies $[3,11]$.

We also observe that the performance of the two production GTM systems are quite similar. This is mostly due to the artifacts of using PlanetLab nodes as the clients - most PlanetLab nodes are deployed in universities and research institutes, and tend to co-locate with their LDNSes. In this sense, PlanetLab nodes are a very biased population to evaluate the client-LDNS mismatch problem. To compensate, in the following experiment, we create an adversary clientLDNS mismatch scenario by switching the clients from their original LDNSes to public DNS systems, which are known to be further away [6]. ${ }^{5}$

\subsection{Resilience to LDNS Change}

Instead of using its original LDNS, a client can make local configurations to use public DNS systems (such as Google, Level3 and OpenDNS) as its LDNS. We have monitor the public DNS systems for more than one year, through a flight

\footnotetext{
${ }^{5}$ An advanced system [18], which measures the latency between data center and LDNS and selects the best data center using the measurement, would perform much better than the any-cast based production systems. Nevertheless, it would still suffer if LDNS is no longer co-located with client.
}

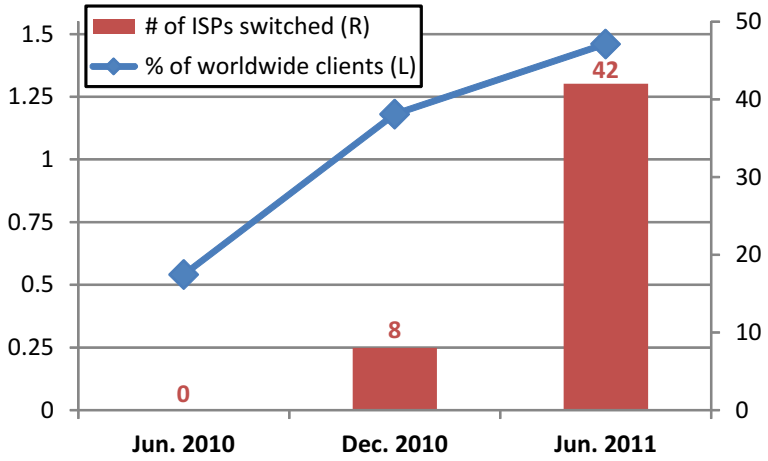

Figure 4: Evolution of Google Public DNS

experiment which correlates clients with their LDNS [6]. The experiment allows us to find which clients are using which public DNS systems as their resolver. We repeated the experiment three times, each half-year apart: in June 2010, December 2010 and June 2011. Figure 4 reports the results of the clients that use Google Public DNS. We find that the percentage of clients has tripled from $0.5 \%$ to $1.5 \%$. In addition, the number of ISPs that switched to Google Public DNS increased from 0 to 42 in just a year. We use the following heuristics: $i$ ) only ISPs (really ASes) with at least 500 unique client IP addresses are considered; $i i$ ) when more than $90 \%$ of its clients use Google Public DNS as their resolver, an ISP is declared to be using Google Public DNS. This clears show that public DNS systems are gaining traction.

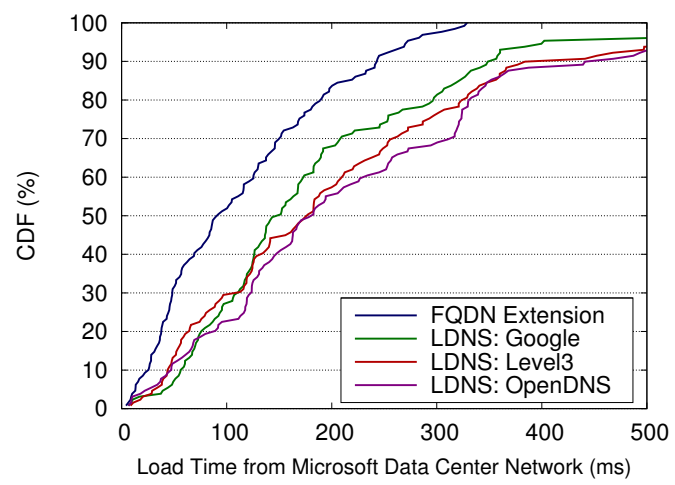

Figure 5: Resilience to LDNS Change

With conventional GTM solutions, intuitively, when a client switches from its original LDNS to a public DNS system, its performance will be further degraded. On the other hand, the FQDN extension solution is independent of LDNS and therefore not affected by such switching at all.

To this end, we have conducted additional experiments to evaluate the impact of switching from LDNS to public DNS systems and the resilience of FQDN extension under such switching. We configure each client to use Google, Level3 and OpenDNS as its LDNS and again download the test object from the best data center determined by the Anycast GTM by DNS ${ }^{6}$.

Figure 5 shows the load time comparison between FQDN extension and switching from LDNS to the three public DNS

${ }^{6}$ Anycast GTM by Client is not affected by such switching. But again, anycast itself leads to suboptimal selection. 
systems. We observe that the switching indeed degrades the performance. The median load time now rises to 152 $\mathrm{ms}, 178 \mathrm{~ms}$ and $183 \mathrm{~ms}$, respectively, when using Google, Level3 and OpenDNS. In addition, the 95-percentile load time now rises to $402 \mathrm{~ms}, 582 \mathrm{~ms}$ and $582 \mathrm{~ms}$, respectively. In short, the savings of FQDN extension are $32 \%, 53 \%$ and $53 \%$, respectively - even more significant reduction!

\section{DISCUSSIONS}

\subsection{Deployment}

FQDN extension can be deployed immediately for many online services without modifying either client or local resolver. The deployment can be piggy-backed on existing client-side URL-rewriting mechanism. Let's use Web search as to illustrate a concrete example. When a user searches "Microsoft Research" on Bing, the first result link appears to point to the right URL http://research.microsoft.com. When the user clicks the link, however, the real URL loaded by the browser is actually http://www.bing.com/$\mathrm{fd} / \mathrm{ls} / \mathrm{GLinkPing}$. aspx?IG=..., which is then redirected to Microsoft Research. Behind the scene, Bing uses JavaScript to intercept the clicked link and re-write it to the actual loaded URL. In this case, FQDN extension can be easily implemented by pre-appending the GTM ID and re-writing the URL to http://gtm-id.www.bing.com/... instead. It is conceivable that the same JavaScript can be used to retrieve GTM ID in the background, even before the user clicking the link.

In long term, FQDN extension can be realized through a client-side change, such as Web browser modification. A Web service may indicate its support of FQDN extension by including a new HTTP header in its response, where the interface to retrieve GTM ID is also specified. A compliant Web browser will retrieve GTM ID from the interface, refresh GTM ID upon expiration, augment hostname and performance resolution based on the augmented hostname.

\subsection{Latency and Overhead}

Retrieving and refreshing GTM ID incur latency and overhead. Fortunately, since it represents the cluster a client belongs to, GTM ID should not change frequently. For instance, if IP prefix is used as GTM ID, then it only needs to refresh when the client IP address changes. Therefore, we expect the frequency of refreshing GTM ID in the order of hours, instead of seconds - the TTL of the resolution result of www.bing.com is merely 20 seconds.

\subsection{Practicality Concerns}

The FQDN extension solution should not cause privacy concern. No matter whether GTM ID is generated by an Internet service, by third parties (such as CDNs) or by an ISP, the IP address of the client is already exposed as long as the client accesses the service. Having the client contacting GTM directly does not expose additional identifying information.

The solution will inevitably increase LDNS cache size. Different clients behind the same LDNS could augment same FQDN with different GTM IDs and thus cause many augmented FQDNes cached at the LDNS for the same service. The increase will be similar to adopting the existing proposal of modifying LDNS [8].

Finally, we comment that the solution is not applicable when clients connecting through mobile networks, where even their IP addresses, let alone the derived GTM IDs, carry insufficient information for making GTM decision.

\section{CONCLUSION}

In this paper, we describe the client-LDNS mismatch problem. We review the existing proposals to address the problem and analyze their limitations. We then summarize the requirements for an ideal solution.

We propose a simple new solution, named "FQDN extension", to address the client-LDNS mismatch problem. We build a prototype system and demonstrate substantial gain. Using JavaScript, the solution can be deployed immediately for some online services, such as Web search, without modifying either client or local resolver.

\section{REFERENCES}

[1] AWS CloudFront Resources http://aws.amazon.com/cloudfront/\#resources.

[2] M. TariQ, A. Zeitoun, V. Valancius, N. Feamster, and M. Ammar, Answering What-If Deployment and Configuration Questions with WISE. ACM Sigcomm (2008).

[3] C. Huang, Y. A. Wang, J. Li, and K. W. Ross, Measuring and Evaluating Large-Scale CDNs. Microsoft Research Technical Report MSR-TR-2008-106 (2008).

[4] Z. M. Mao, C. Cranor, F. Douglis, M. Rabinovich, O. Spatscheck, AND J. WANG, A Precise and Efficient Evaluation of the Proximity between Web Clients and their Local DNS Servers. USENIX ATC (2002).

[5] A. Shaikh, R. Tewari, and M. Agrawal, On the Effectiveness of DNS-based Server Selection. IEEE INFOCOM (2006).

[6] C. Huang, D. A. Maltz, A. Greenberg, and J. Li, Public DNS Systems and Global Traffic Management. IEEE INFOCOM (2011).

[7] R. Kohavi, R. M. Henne, and D. Sommerfield, Practical Guide to Controlled Experiments on the Web: Listen to Your Customers not to the Hippo. ACM KDD (2007).

[8] C. Contavalli, W. van der Gaast, S. Leach, and D. Rodden, Client IP information in DNS Requests. IETF Internet Draft draft-vandergaast-edns-client-ip-01 (2010).

[9] M. Andrews, B. Shepherd, A. Srinivasan, P. Winkler, and F. Zane, Clustering and Server Selection Using Passive Monitoring. IEEE INFOCOM (2002).

10] R. Krishnan, H. V. Madhyastha, S. Srinivasan, S. Jain, A. Krishnamurthy, T. Anderson, and J. Gao, Moving Beyond End-to-End Path Information to Optimize CDN Performance. ACM IMC (2009).

[11] H. Ballani, P. Francis, and S. Ratnasamy, Measurementbased Deployment Proposal for IP Anycast. ACM IMC (2006).

[12] C. Partridge, T. Mendez, and W. Milliken, RFC1546: Host Anycasting Service, (1993).

[13] M. Stemm, R. Katz, and S. Seshan, A Network Measurement Architecture for Adaptive Applications. IEEE INFOCOM (2000).

[14] V. K. Adhikari, S. Jain, And Z. Zhang, YouTube Traffic Dynamics and Its Interplay with a Tier-1 ISP: An ISP Perspective. ACM IMC (2010).

[15] SPDY: An Experimental Protocol for a Faster Web. Google Whitepaper (2009)

[16] A. Pathak, Y. A. Wang, C. Huang, A. Greenberg, Y. C. Hu, R. Kern, J. LI, AND K. W. Ross, Measuring and Evaluating TCP Splitting for Cloud Services. PAM (2010).

[17] N. Dukkipati, T Refice, Y. Cheng, J. Chu, N. Sutin, A. Agarwal, T. Herbert and A. Jain, An Argument for Increasing TCP's Initial Congestion Window. ACM CCR (2010).

[18] C. Huang, N. Holt, Y. A. Wang, A. Greenberg, J. Li and K. W. Ross, A DNS Reflection Method for Global Traffic Management. USENIX ATC (2010).

[19] B. Ager, W. Muhlbauer, G. Smaragdakis and S. Uhlig, Comparing DNS Resolvers in the Wild. ACM IMC (2010).

[20] C. Kreibich, N. Weaver, B. Nechaev and V. Paxson, Netalyzr: Illuminating the Edge Network. ACM IMC (2010).

[21] B. Krishnamurthy and J. Wang, On Network-Aware Clustering of Web Clients. ACM SIGCOMM (2002).

[22] I. Poese, B. Frank, B. Ager, G. Smaragdakis and A. Feldmann, Improving Content Delivery using Provider-aided Distance Information. ACM IMC (2010). 